\title{
ITS and pB2.5 gene expression of Naegleria fowleri in drug resistance
}

\author{
Jundee Rabablert ${ }^{1}$, Supathra Tiewcharoen ${ }^{2 *}$, Virach Junnu ${ }^{2}$ \\ ${ }^{1}$ Department of Biology, Faculty of Science, Silpakorn University, Nakhon Pathom, Thailand; \\ ${ }^{2}$ Department of Parasitology, Faculty of Medicine Siriraj Hospital, Mahidol University, Bangkok, Thailand; \\ *Corresponding Author: sistc@,mahidol.ac.th
}

Received 8 May 2011; revised 22 June 2011; accepted 13 July 2011.

\begin{abstract}
Naegleria fowleri was causative agent of primary amoebic meningoencephalitis (PAM). Accroding to the failure of treatment, several researches reported the activity of chemotherapeutic drugs against $\boldsymbol{N}$. fowleri but we did not know the drug resistance of the amoebae. The purpose of this study was to examine the effects of drugs (amphotericin B, artesunate, azithromycin, voriconazole, chlorpromazine, fluconazole and gentamicin sulphate) on ITS and pB2.3 genes of Naegleria fowleri trophozoites. Our study demonstrated gene expression of treated $N$. fowleri by RT-PCR. The results reviewed that ITS gene of $N$. fowleri showed up regulate to amphotericin $B$, azithromycin and gentamicin sulphate, while pB2.3 gene showed up regulate to artesunate. These results compared with beta actin (house keeping gene) expression at time intervals $15-120 \mathrm{~min}$. The change of gene expression of treated N.fowleri was possibly to cause of drug resistance. The mechanism of drug resistance genes ITS and pB2.3 of $N$. fowleri should be clarified in further study.
\end{abstract}

Keywords: Naegleria Fowleri; ITS; pB2.3; Drug Resistance

\section{INTRODUCTION}

Naegleria fowleri causes severe meningoencephalitis mainly in children and young adults. Due to the treatments have not been succeeded, most of patients die from $N$. fowleri infection [1]. The effect of drug against $N$. fowleri has been carried out both in vitro and in vivo studies which provided for clinical trends for treatment [2]. A prelude of drug resistant has been frequently documented in recent years [3]. Several researches on antifungal resistant have been focused on elucidating the molecular basis and transcriptional regulation of azole in
Candida spp. [4]. The resistance to azole uptake of $C$. albicans can be achieved with the introduction of key point mutation in and/or up regulation of gene expression which encodes on efflux pump; EGR11, MDR including ATP-binding cassette transporter molecules $C D R 2$ [5]. In addition, antifungal resistant was involved sterol uptake which was controled by UPC2 gene [6]. Owing to failure treatment of PAM, one of the major problems was drug resistant from gene alteration. Up to date, a study has been addressed the intrinsic resistant on $n f a 1$ and $\mathrm{Mp} 2 \mathrm{Cl} 5$ genes which regulated pathogenesis of the amoebae. The results demonstrated that either nfa1 resistant to fluconazole or $\mathrm{Mp} 2 \mathrm{Cl} 5$ resistant to amphotericin $\mathrm{B}$, azithromycin and artesunate of $N$. fowleri were found [7]. Owing to the dominant ITS, located in the 5.8S rRNA gene and species-specific chromosomal DNA $p B 2.3$ genes were used for identify pathogenic $N$. fowleri [8] and diversity of $N$. fowleri at molecular level [9], we investigated the activity of drugs on ITS and $p B 2.3$ genes of $N$. fowleri. This report revealed the ability of Naegleria genes against drugs of choice.

\section{MATERIALS AND METHODS}

\subsection{Naegleria Fowleri Cultivation}

Free living $N$. fowleri trophozoites (Khon-Kaen strain) were cultured in Nelson's medium supplemented with $5 \%$ heat-inactivated fetal calf serum (FCS) without antibiotics at $37^{\circ} \mathrm{C}$. Trophozoites were tested with the concentration of amphortericin B, voriconazole, fluconazole, chlorpromazine, artesunate, azithromycin and gentamicin at $\mathrm{IC}_{50}$ [10] during 15 - $120 \mathrm{~min}$, triplicate. Untreated trophozoite was used for negative control. At indicated times, trophozoites were twice washed with normal saline and frozen at $-80^{\circ} \mathrm{C}$ until required.

\subsection{RNA Extraction}

Total RNA was extracted from untreated or treated 
amoebae trophozoites using Tri Reagent (Sigma-Aldrich, USA). For positive control, nf actin gene (housekeeping gene) of $N$.fowleri was confirmed by primer $5^{\prime \prime}$ - ACT CTG GTG ATG GTG TCT CTC ACA C- $3^{\prime}$ and $5^{\prime \prime}-$ CTC TGA CAA TTT CTC TCT CAG TGG-3'. The amplicons of amoebae were prepared from primers of ITS (ITS1; 5'-GAACCTGCGTAGGGATCATTT-3' and ITS2; 5'TTTCTTTTCCTCCC CTTATTA-3') and pB2.3 (p3f; 5'GTGAAAACCTTTTTTCCATTTACA-3') and p3r; 5'AAATAAAAA TTACCATTTGAAA-3') by one - step Super-script PCR (Invitrogen, Gransland). MW of each amplicon was detected by $1.5 \%$ Agarose Gel Electrophoresis at $100 \mathrm{~V}$ for $30 \mathrm{~min}$.

\section{RESULTS AND DISCUSSION}

In our studies we demonstrated the responsibility of $N$. fowleri to the effects of drugs (amphotericin B, artesunate, azithromycin, voriconazole, chlorpromazine, fluconazole and gentamicin sulphate) on ITS and of pB2.3 gene by RT-PCR. The number of $N$. fowleri treated or untreated $N$. fowleri was not significant different between two groups at time intervals (the results was not shown) $(\mathrm{t}<0.005)$. Total RNA was extracted from this two groups using Tri Reagent (Sigma-Aldrich, USA) at indicated time. As a positive control, we used $n f$ actin to amplify under the same RT-PCR condition and regulation of expression of $n f$ actin gene was detected at 170 $\mathrm{bp}$ at $15-120 \mathrm{~min}$. The up regulate of $n f$ actin was compared with treated or untreated amoebae at every point of time. We found that untreated N.fowleri showed up regulate of ITS gene at 450 while ITS gene expression from treated trophozoites with voriconazole, fluconazole or chlorpromazine was not observed during $120 \mathrm{~min}$. In contrast, trophozoites treated with amphotericin B was found at least 30 min whereas trophozoites treated with azithromycin or gentamicin was shown at least $45 \mathrm{~min}$ (Figure 1). Similarly, we tested the drugs activity against $p B 2.3$ gene expression of amoebae trophozoites. The untreated trophozoites showed bright band fragment at $310 \mathrm{bp}$ as shown in Figure 2. Interesting, we did not observe $p B 2.3$ gene expression from treated trophozoites with a panel of drugs at $120 \mathrm{~min}$, except artesunate. It is possibly suggested that ITS gene of $N$. fowleri trophozoite was resisted to amphotericin $\mathrm{B}$, azithromycin or gentamicin including the $p B 2.3$ gene was resisted to artesunate.

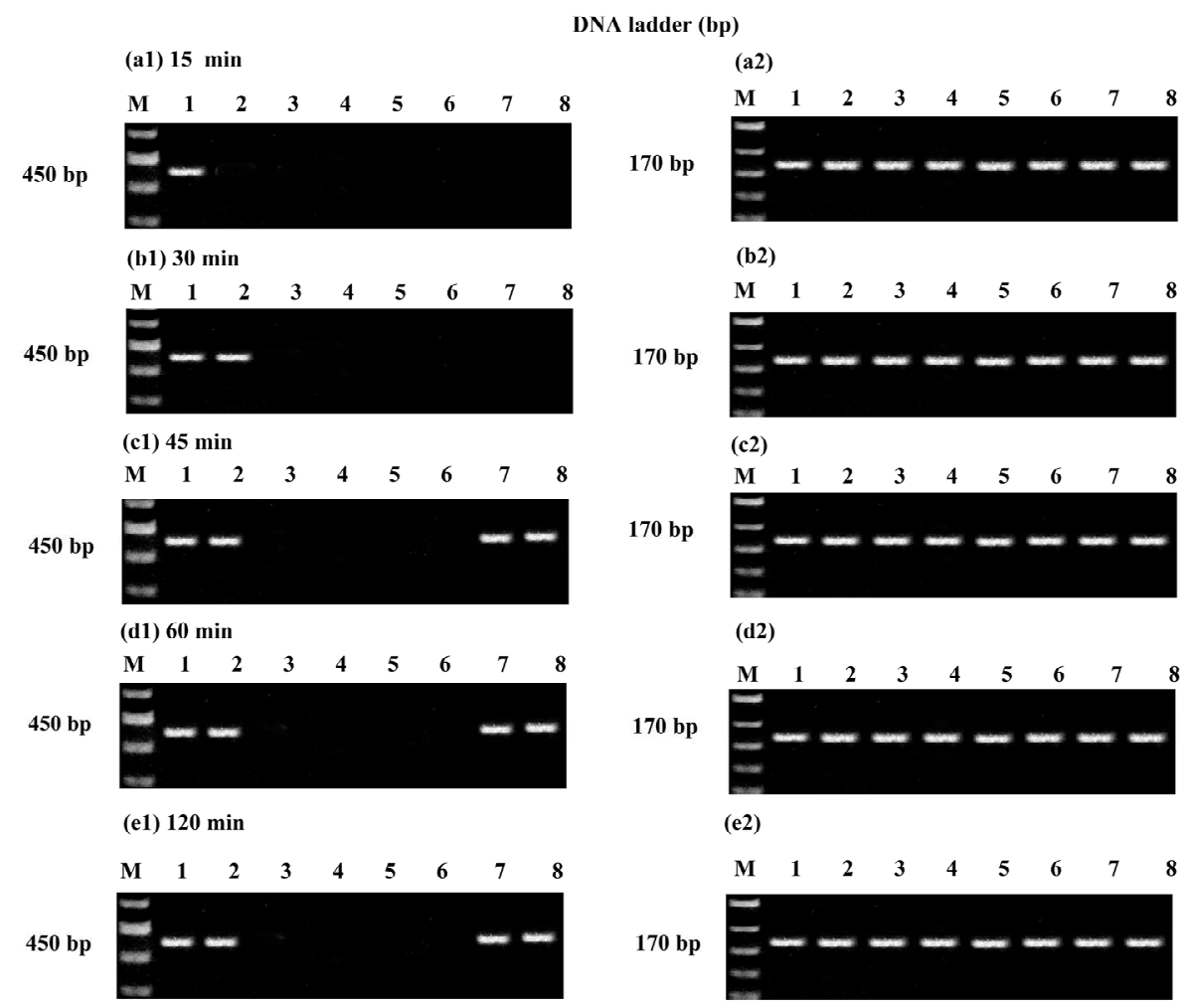

Figure 1. Effect of drugs on ITS gene of $N$. fowleri by RT-PCR at 15, 30, 45, 60 and 120 min (a1-e1) compared with the positive expression control, nf actin gene at the same time (a2-e2). Untreated N. fowleri showed bright band fragment at $450 \mathrm{bp}$ (lane1). Treated $N$. fowleri with amphotericin B (lane 2), voriconazole (lane 3), fluconazole (lane 4), chlorpromazine (lane 5), artesunate (lane 6), azithromycin (lane 7), and gentamicin sulphate (lane 8), respectively. 
(a) $15 \mathrm{~min}$ DNA ladder (bp)

(f1) $15 \mathrm{~min}$

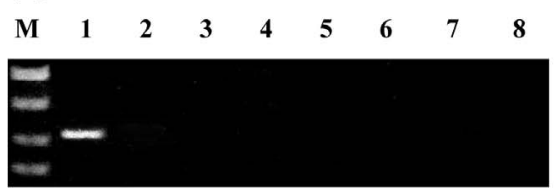

(g1) $30 \mathrm{~min}$

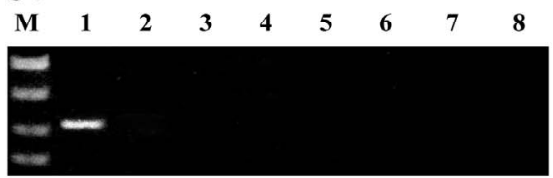

(h1) $45 \mathrm{~min}$

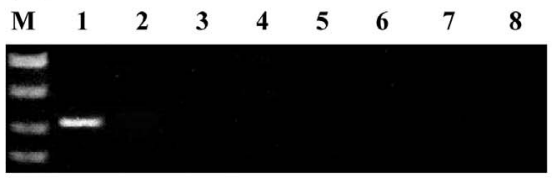

(i1) $60 \mathrm{~min}$

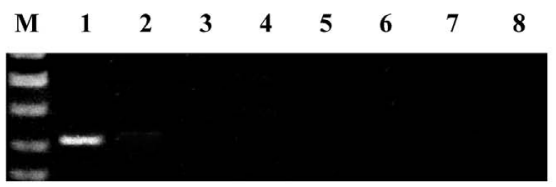

(j1) $120 \mathrm{~min}$

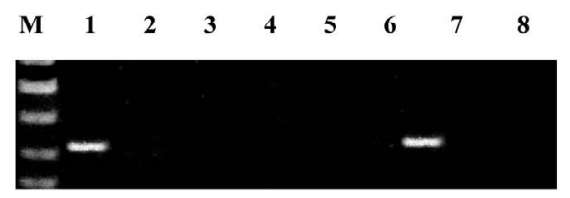

(f)

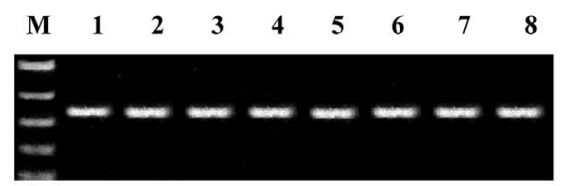

(g2)

$170 \mathrm{bp}$

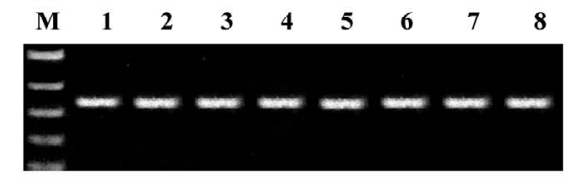

(h2)

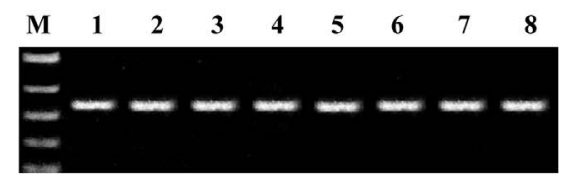

(i2)

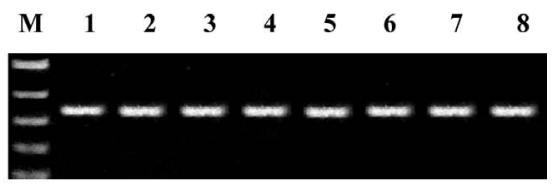

(j2)

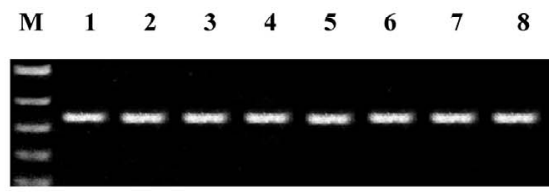

Figure 2. Effect of drugs on $p B 2.3$ gene of $N$. fowleri by RT-PCR at 15, 30, 45, 60 and 120 min (f1-j1) compared with the positive expression control, nf actin gene(170) at the same time (f2-j2). Untreated N. fowleri showed bright band fragment at $310 \mathrm{bp}$ (lane1). Treated $N$. fowleri with amphotericin B (lane 2), voriconazole (lane 3), fluconazole (lane 4), chlorpromazine (lane 5), artesunate (lane 6), azithromycin (lane 7), and gentamicin sulphate (lane 8), respectively.

Amphotericin B has been generally recognized for fungal and protozoa treatment, many publications reported amphotericin B-resistant genes in Candida lusitaniae, Saccharomyces cerevisiae [11] and Cryptococcus neoformans [12]. However, it has been reported in pathogenic protozoa; Entamoeba histolytica, Giardia lamblia, Trichomonas vaginalis and also occurred anaerobic protozoa; Blastocystis hominis, Cryptosporidium parvum, Isospora spp., Cyclosporssporidia spp. [13]. In addition, amphotericin B resistant gene was also found in vector born protozoa, Leishmania tarentolae [14].

The mechanism of amphotericin B, polyene resistant of C. albicans and S. cerevisiae caused by mutation of $E G R$ genes which control the production of ergosterol and sensitivity to polyenes. As the result of EGR6 mutant strain of $C$. lusitaniae transcription, the reduced ergosterol content was appeared [15]. A study of EGR6 mutant caused unable to form amphotericin B-generated pores in the cell membrane on Candida spp [16]. Our study revealed amphotericin B resistant ITS gene of $N$. fowleri was firstly appeared. Moreover, drug resistant; azithromycin, gentamicin sulphate to ITS and artesunate to $p B 2.3$ of $N$. fowleri were also demonstrated. A few publications reported the azithromycin resistant in $\mathrm{Pseu}$ domonas aeruginosa [17] and genetic mutation at 23S rRNA region of Ureaplasma urealyticum and Neisseria gonorrhoeae [18]. Gene resistant, ermG , to azithromycin was established in Bacteroides. Mechanism of azithromycin resistant established at MexCD-OprJ pump of Pseudomonas aeruginosa biofims [19].

Gentamicin has been general used in cultivation; Enterococcus faecali [20], Pseudomonas aeruginosa [21]. The occurrence of gentamicin-resistant genes of gram negative bacteria; Enterobacteriaceae, Pseudomonas, Acinetobacter were isoloated from different environment mainly originating from sewage, faces, coastal water 
polluted with wastewater and the distribution of these resistant genes could broadly transfer to hosts [22]. Gentamicin-resistant to $N$. fowleri has been found in vitro since 2009 [10].

This study showed that gentamicin resistant concerned both gene expression and aminoglycoside activity [20]. Artesunate was used to treatment for falciparum malaria due to artesunate accumulated lipids bodies and induced oxidative membrane damage [23]. Furthermore, it blocked protein synthesis of yeast cells [24] and inhibited replication of cytomegalovirus [25]. According to general use of artesunate, the artemisinin resistance to malaria was found in clinical trial [26]. The resistance gene $m d r 1$, cg10, tctp, and atp6 to artemisinin of Plasmodium chabaudi chabaudi were developed and transmitted to its derivatives [27]. The $p B 2.3$ resistant gene of $N$. fowleri was appeared, thereby it located in mitochondria and chromosome of the amoebae. In conclusion, one of the drug treatment failure focused on ITS and $p B 2.3$ genes of N. fowleri.

\section{ACKNOWLEDGEMENTS}

This work was partially suppoted by: grant Number RGP 2552/02 from Department of Biology, Faculty of Science, Silpakorn University at Sanamchan Palace, Nakhon Pathom, Thailand. We thank the head of Department Parasitology, Faculty of Medicine Siriraj hospital, Mahidol University for provided the research facility.

\section{REFERENCES}

[1] Hebbar, S., Bairy, I., Bhaskaranand, N., Upadhyaya, S., Sarma, M.S., Shetty, A.K.(2005) Fatal case of Naegleria fowleri meningo-encephalitis in an infant: Case report. Annals of Tropical Paediatrics, 25, 223-226. doi:10.1179/146532805X58166

[2] Soltow, S.M. and Brenner, G.M. (2007) Synergistic activities of azithromycin and amphotericin B against Naegleria fowleri in vitro and in a mouse model of primary amebic meningoencephalitis. Antimicrobial Agents and Chemotherapy, 51, 23-27. doi:10.1128/AAC.00788-06

[3] Donadio, S., Maffioli, S., Monciardini, P., Sosio, M. and Jabes, D. (2010) Antibiotic discovery in the twenty-first century: current trends and future perspectives. The Journal of Antibiotics, 63, 423-430.

doi:10.1038/ja.2010.62

[4] Barker, K.S. and Rogers, P.D. (2006) Recent insights into the mechanisms of antifungal resistance. Current Infectious Disease Reports, 8, 449-456. doi:10.1007/s11908-006-0019-3

[5] Akins, R.A. (2005) An update on antifungal targets and mechanisms of resistance in Candida albicans. Medical Mycology, 43, 285-318. doi:10.1080/13693780500138971

[6] Vermitsky, J.P. and Edlind, T.D. (2004) Azole resistance in Candida glabrata: Coordinate upregulation of mul- tidrug transporters and evidence for a Pdr1-like transcription factor. Antimicrobial Agents and Chemotherapy, 48, 3773-3781. doi:10.1128/AAC.48.10.3773-3781.2004

[7] Tiewcharoen, S., Rabablert, J., Worawirunwong, D., Pratumsrikajorn, T., Limsangurai, S. and Junnu, V. (2011) Activity of chlorpromazine on $n f a 1$ and Mp2CL5 genes of Naegleria fowleri trophozoites. Health, 3, 166-177. doi:10.4236/health.2011.33032

[8] Robinson, B.S, Monis, P.T. and Dobson, P.J (2006) Rapid, sensitive, and discriminating identification of Naegleria spp. by real-time PCR and melting-curve. Applied and Environmental Microbiology, 72, 5857-5863. doi:10.1128/AEM.00113-06

[9] Tsvetkova, N., Schild, M., Panaiotov, S., et al. (2004) The identification of free-living environmental isolates of amoebae from Bulgaria. Parasitology Research, 92, 405413.

[10] Tiewcharoen, S., Rabablert, J. and Junnu, V. (2009) In vitro susceptibility of Naegleria fowleri trophozoites to, amphotericin B-combined chlorpromazine. Research Journal of Microbiology, 4, 320-333. doi:10.3923/jm.2009.320.333

[11] Zhang, L., Zhang, Y., Zhoul, Y., et al. (2002) Response of gene expression in Saccharomyces cerevisiae to amphotericin B and nystatin measured by microarrays. Journal Antimicrobial Chemotheapy, 49, 905-915. doi:10.1093/jac/dkf001

[12] Manfredi, R., Fulgaro, C., Sabbatani, S., Legnani, G. and Fasulo G. (2006) Emergence of amphotericin B-resistant Cryptococcus laurentii meningoencephalitis shortly after treatment for Cryptococcus neoformans meningitis in a patient with aids. Aids Patient Care and STDs, 20, 227-232. doi:10.1089/apc.2006.20.227

[13] Orozco, E., Marchat, L.A, Gómez, C., López-Camarillo, C. and Pérez, D.G. (2009) Drug resistance mechanisms in Entamoeba histolytica, Giardia lamblia, Trichomonas vaginalis, and opportunistic anaerobic protozoa. Antimicrobial Drug Resistance Infectious Disease, F, 549-559.

[14] Singh, A.K, Papadopoulou, B., Ouellette, M (2009) Gene Amplification in Amphotericin B-Resistant Leishmania tarentolae. Experimental Parasitology, 99, 141-147. doi:10.1006/expr.2001.4663

[15] O'Shaughnessy, E.M, Lyman, C.A. and Walsh, T.J. (2009) Amphotericin B: Polyene resistance mechanisms. Antimicrobial Drug Resistance Infectious Disease, D, 295305.

[16] Mandell, G.L. and Coleman, E.J. (2000) Activities of antimicrobial agents against intracellular pneumococci. Antimicrobial Agents and Chemotherapy, 44, 2561-2563. doi:10.1128/AAC.44.9.2561-2563.2000

[17] Mulet, X., Maciá, M.D., Mena, A., Juan, C., Pérez, J.L., Oliver, A. (2009) Azithromycin in Pseudomonas aeruginosa biofilms: Bactericidal activity and selection of $n f x B$ mutants. Antimicrobial Agents and Chemotherapy, 53, 1552-1560. doi:10.1128/AAC.01264-08

[18] Galarza, P.G., Abad, R., Canigia, L.F, et al. (2010) New mutation in $23 \mathrm{~S}$ rRNA gene associated with high level of azithromycin resistance in Neisseria gonorrhoeae. Antimicrobial Agents and Chemotherapy, 54, 1652-1653.

[19] Gillis, R.J., White, K.G., Choi, K.H., et al. (2005) Molecular basis of azithromycin-resistant Pseudomonas aeruginosa biofilms. Antimicrobial Agents and Chemo- 
therapy, 49, 3858-3867.

doi:10.1128/AAC.49.9.3858-3867.2005

[20] Chow, J.W. (2000) Aminoglycoside resistance in enterococci. Clinical Infectious Diseases, 31, 586-589. doi:10.1086/313949

[21] Livermore, D.M. (2002) Multiple mechanisms of antimicrobial resistance in Pseudomonas aeruginosa: Our worst nightmare? Clinical Infectious Diseases, 34, 634640. doi: $10.1086 / 338782$

[22] Heuer, H., Krögerrecklenfort, E., Wellington, E.M., et al. (2002) Gentamicin resistance genes in environmental bacteria: Prevalence and transfer. FEMS Microbioogy Ecology, 42, 289-302.

[23] Hartwig, C.L, Rosenthal, A.S, D'Angelo, J., et al. (2009) Accumulation of artemisinin trioxane derivatives within neutral lipids of Plasmodium falciparum malaria parasites is endoperoxide-dependent. Biochemistry Pharmacology, 77, 322-336. doi:10.1016/j.bcp.2008.10.015

[24] Li, W., Mo, W., Shen, D., et al. (2005) Yeast model un- covers dual roles of mitochondria in the action of artemisinin. PLoS Genetics, 1, 329.

doi:10.1371/journal.pgen.0010036

[25] Kaptein, S.J., Efferth, T., Leis, M., et al. (2006) The anti-malaria drug artesunate inhibits replication of cytomegalovirus in vitro and in vivo. Antiviral Research, 69, 60- 69. doi:10.1016/j.antiviral.2005.10.003

[26] Meshnick, S.R. (2002) Artemisinin: Mechanisms of action, resistance and toxicity. International Journal for Parasitology, 32, 1655-1660. doi:10.1016/S0020-7519(02)00194-7

[27] Afonso, A., Hunt, P., Cheesman, S., et al. (2006) Malaria parasites can develop stable resistance to artemisinin but lack mutations in candidate genes atp6 (Encoding the sarcoplasmic and endoplasmic reticulum $\mathrm{Ca}^{2+}$ ATPase), tctp, mdr1, and cg10. Antimicrobial Agents and Chemotherapy, 50, 480-489.

doi:10.1128/AAC.50.2.480-489.2006 